\title{
Contralateral and ipsilateral disorders of visual attention in patients with unilateral brain damage
}

\author{
Guido Gainotti, Laura Giustolisi, Ugo Nocentini
}

\section{Abstract}

To explain the prevalence of unilateral spatial neglect in patients with right brain damage, Heilman et al have suggested that the attentional neurons of the right parietal lobe might have bilateral receptive fields, whereas the homologous cells of the left hemisphere would have strictly contralateral receptive fields. One implication of this theory is that patients with right brain damage should show a prevalence of disorders of visual attention not only in the half space contralateral to the damaged hemisphere, but also in the ipsilateral one. To check this theory, 50 control subjects, 102 right and 125 left brain-damaged patients were given a drawing completion task in which patients were requested to complete the missing parts of a star, a cube and a house. Omissions of lines lying on the sides of the models contralateral and ipsilateral to the damaged hemisphere were taken separately into account. Results did not confirm the hypothesis, since right brain-damaged patients failed to complete the contralateral sides of the models much more frequently than patients with left brain injury, but no difference was found between the two hemispheric groups when ipislateral disorders of visual attention were taken into account. Furthermore, no correlation was found between omissions of lines lying on the sides of the models contralateral and ipislateral to the damaged hemisphere. This finding suggests that contralateral and ipislateral disorders of visual attention are not due to the same mechanism in right brain-damaged patients. The alternative hypothesis viewing ipsilateral disorders as resulting from a widespread lowering of general attention (and only contralateral neglect reflecting a specific disorder of visual attention) was supported by results obtained on a verbal memory test, used to evaluate the general cognitive and attention level of the patients. Patients with clear-cut ipislateral inattention obtained very low scores on this test, whereas patients with severe contralateral neglect, but not ipislateral inattention scored within the normal range on the verbal memory test.

Since the classic observations of Brain, ${ }^{1}$ who first stressed the links existing between uni- lateral spatial neglect and right hemisphere lesions, several clinical and experimental investigation $\mathrm{s}^{2-8}$ have confirmed that unilateral neglect is significantly more frequent and severe in right than in left brain damaged patients. Heilman et $a l^{9-11}$ have tried to explain this hemispheric asymmetry by assuming that the attentional neurons of the right parietal cortex may have bilateral receptive fields, whereas the homologous cells of the left parietal cortex would have strictly contralateral receptive fields. According to this theory, left hemisphere injury would rarely produce contralateral neglect because the intact cells of the right parietal lobe can deal with both contralateral and ipsilateral stimuli. On the contrary, right hemisphere damage would often produce contralateral neglect because the intact neurons of the left parietal lobe cannot deal with stimuli presented in the left (ipsilateral) visual field.

One implication of this model is that right hemisphere injury should produce not only contralateral neglect but also some degree of inattention for the ipsilateral half space, whereas no ipsilateral inattention should be observed in patients with left brain damage.

These predictions have been recently confirmed by Weintraub and Mesulam ${ }^{12}$ on a shape cancellation task and on a manual exploration task performed without visual control, but not by Gainotti et $\mathrm{al}^{13}$ on a test of double tactile simultaneous stimuli of different parts of the body. In both studies contralateral neglect was more frequent and severe in right than in left brain damaged patients, but ipsilateral inattention following right brain injury was observed by Weintraub and Mesulam ${ }^{12}$ but not by Gainotti et al. ${ }^{13}$ Two different interpretations can be put forward to explain these conflicting results: a) according to the first interpretation, the double tactile stimulation paradigm used by Gainotti et al $^{13}$ does not adequately test Heilman's hypothesis, either because the extinction phenomenon considered in their research must be set apart from hemi-inattention or because Heilman's model holds for the visual but not for the tactile modality; b) according to the second interpretation, ipsilateral disorders of visual attention observed by Weintraub and Mesulam ${ }^{12}$ in patients with right brain damage could be explained by factors other than an impairment of parietal attentional neurons with bilateral or ipsilateral receptive fields.

Gainotti et al ${ }^{13}$ observed for example that patients with severe left sided neglect often 
limit their attention to the extreme right part of extrapersonal space. Therefore, most errors performed on the right (ipsilateral) side of the sheet during the cancellation test used by Weintraub and Mesulam ${ }^{12}$ could be due to an extension of contralateral neglect beyond the midline, rather than to an independent ipsilateral hemi-inattention.

Furthermore, the same authors noted that their patients features of ipsilateral tactual extinction were significantly related to non specific factors (such as old age, severity of cerebral lesion or widespread mental impairment) which could reduce the general attentional resources of the subjects. They, therefore, argued that ipsilateral attentional disorders observed in patients with right brain damage could be due to a widespread attentional defect and not to a property spatial disorder of attention, as hypothesised by Heilman's theory.

To check these alternative interpretations, we have designed a study in which disorders of visual attention were investigated in the half space contralateral and ipsilateral to the damaged hemisphere with two methodological cautions: a) to consider as indicative of properly ipsilateral inattention, only the omissions of elements lying on the extreme lateral parts of a pattern, as this avoids the danger of attributing to ipsilateral inattention the consequences of a contralateral neglect extending beyond the midline; b) to take into account two different factors which could produce defects of visual attention on the half space ipsilateral to a brain lesion: 1) specific factors, linked to the bilateral receptive fields of attentional parietal neurons; 2) non specific factors, which could reduce the attentional resources of the subject and hence produce generalised disorders of attention.

\section{Material and methods \\ Patients}

Two groups of patients with left $(N=125)$ or right $(\mathrm{N}=102)$ brain damage and 50 normal controls were examined. Although patients were unselected, the following criteria of exclusion from the study were retained: presence of obvious clinical signs of widespread mental impairment or of bilateral brain damage; less than five years of formal education; left handedness. The vascular patients were examined after the acute post-ictal stage and the neoplastic patients were studied after surgery or when signs of brain oedema had disappeared.

Figure Examples of a star stimulus and of a cube stimulus. Patients were requested to complete the missing lines of each stimulus; only lines lying on the external left $(L)$ or right $(R)$ sides of the models were taken into account to evaluate contralateral and ipsilateral disorders of visual attention. Lines concerning central (or internal) parts of the figures (NC) were not considered.
Controls were affected by psychoneurosis or by peripheral nerve disorders and were matched to the patients with brain damage for age and education. No significant differences were found between right and left brain damaged patients for age, educational level and cause of illness.

Drawing completion task used to evaluate contralateral and ipsilateral disorders of visual attention

A drawing completion task, in which patients were requested to complete the missing parts of a star, a cube, and a house, was considered appropriate to evaluate disorders of visual attention on the half space contralateral and ipsilateral to the brain injury. Two examples of stars, four of cubes and four of houses were used (fig). The missing parts of the figures concerned sometimes the left or the right sides of the models and other times the central parts.

As we wanted to study ipsilateral disorders of attention, by excluding disorders which could be due to an extension beyond the midline of contralateral neglect, we took into account only the omissions of lines tracing the external sides of the models, disregarding those concerning central parts of the drawings. The number of lines to be drawn on the left and right sides of the models was equivalent: 24 on the right and 24 on the left. Each line omitted by the patient on the side of the model ipsilateral or contralateral to the lesion scored 1 point.

Criteria used to assess if ipsilateral omissions on the drawing completion task were due to specific or to non-specific disorders of attention

The nature of the disorders of visual attention detected on the half space ipsilateral to the cerebral lesion was evaluated with reference to criteria intrinsic and extrinsic to the drawing completion task.

The intrinsic criterion consisted in a correlative study of omissions made on the whole test on the sides of the model ipsilateral and contralateral to the brain lesion. It was reasoned that if ipsilateral inattention results from damage to parietal lobe cells with bilateral receptive fields, intermingled with neurons with only contralateral receptive fields, then a strong correlation should be expected between ipsilateral and contralateral disorders of visual attention.

If, on the contrary, only contralateral neglect is due to a specific disorder of visual attention whereas ipsilateral omissions are generically due to a decreased arousal or to reduced attentional resources, then no significant correlation should be found between contralateral and ipsilateral disorders of visual attention.

The extrinsic criterion consisted of: evaluating results obtained by patients with and without ipsilateral disorders of visual attention on a free recall verbal memory task; the Rey's 15 words memory test ${ }^{14}$ which does not tap visual attentional abilities but is highly sensitive to conditions, such as dementia or confusional states, involving a reduction of the general attention resources of the patients. 
The Rey's 15 word memory test

This consists of a list of 15 high frequency semantically unrelated words, which are read aloud by the examiner at a constant rate five consecutive times. The overall number of words recalled after these five consecutive administrations constitutes the immediate recall score, whereas the number of words recalled after a 15 minutes interval, without further repetitions, constitutes the delayed recall score. Since both the immediate and the delayed recall scores are very sensitive to the presence of widespread brain damage ${ }^{15}$ the following predictions were made: a) ipsilateral omissions should be related to poor scores on the Rey's verbal memory test if these omissions are due to a general lowering of the patients' attentional resources; b) on the contrary, no correlations should be observed between verbal memory scores and ipsilateral omissions if the latter disturbance reflects a specific disorder of visual attention.

\section{Results}

A) Incidence of contralateral neglect in patients with right and left brain damage

To obtain a first raw evaluation of the incidence of unilateral neglect in patients with right and left brain damage we considered the following operationally affected by visual hemi-inattention: a) patients with more than one omission on the sides of the drawings contralateral to the lesion, without any omission on the ipsilateral side; b) patients with both contralateral and ipsilateral omissions but with a ratio of 2 to 1 or more between contralateral and ipsilateral omissions. Thirty four right brain-damaged patients out of $102(32.6 \%)$ and six left braindamaged patients out of $126(4.7 \%$ ) were considered as affected by visual hemi-neglect ( $\mathrm{p}<0.001$ ) showing that the "drawing completion task" carefully detects the prevalence of contralateral hemi-inattention in right braindamaged patients which prompted Heilman's hypothesis.

\section{B) Incidence of ipsilateral omissions in patients with right and left brain injury}

Since no control subject made omissions on the

Table 1 Mean number of omissions made by patients with right and left brain damage on the sides of the models contralateral and ipsilateral to the damaged hemisphere

\begin{tabular}{|c|c|c|}
\hline & \multicolumn{2}{|c|}{ Hemispheric side of lesion } \\
\hline & Right & Left \\
\hline \multicolumn{3}{|l|}{ Side of the model } \\
\hline $\begin{array}{l}\text { Ipsilateral } \\
\text { Contralateral }\end{array}$ & $\begin{array}{l}0.49 \\
3 \cdot 24\end{array}$ & $\begin{array}{l}0 \cdot 22 \\
0 \cdot 45\end{array}$ \\
\hline \multicolumn{3}{|l|}{ Two way ANOVA } \\
\hline $\begin{array}{l}\text { Hemispheric group } \\
\text { Side of the model } \\
\text { Interaction }\end{array}$ & $\begin{array}{l}F=30.32 \\
F=28.75 \\
F=20.56\end{array}$ & $\begin{array}{l}\text { p } 0.001 \\
\text { p } 0.001 \\
\text { p } 0.001\end{array}$ \\
\hline \multicolumn{3}{|l|}{ Post-hoc comparisons } \\
\hline $\begin{array}{l}\text { Contralateral omissions } \\
\text { Patients with right versus } \\
\text { left brain damage } \\
\text { Ipsilateral omissions } \\
\text { Patients with right versus } \\
\text { left brain damage }\end{array}$ & \multicolumn{2}{|c|}{$Z=-4 \cdot 48 p \cdot 001$} \\
\hline
\end{tabular}

external lines of models, even a single omission ipsilateral to the side of brain damage was considered as indicative of ipsilateral inattention. Twenty-four right $(23.5 \%)$ and 19 left $(15.0 \%)$ patients with brain damage showed ipsilateral visual inattention according to the above criterion (this difference was not statistically significant).

C) Number of omissions shown by patients with right and left brain damage on the sides of the models contralateral and ipsilateral to the brain lesion

To have a more precise evaluation of the relationships between the hemispheric side of the lesion and disorders of attention for the half space contralateral and ipsilateral to the damaged hemisphere, the number of omissions made by patients with right and left brain damage on the sides of the models contralateral and ipsilateral to the lesion was. calculated. These data were submitted to a two way ANOVA, with two main factors: a) hemispheric side of the lesion and $b$ ) side of the model (contralateral or ipsilateral to the lesion) on which the omission was made. The descriptive statistics and the results of the ANOVA are reported in table 1 . The ANOVA results revealed a significant effect both for the main factors and for their interactions.

Since the distribution of scores did not allow the use of parametric tests, post hoc comparisons were conducted by means of the MannWhitney's $U$ test. The following results were obtained: a) on the sides of the drawings contralateral to the damaged hemisphere the number of omissions was significantly greater in right than in left brain damaged patients; b) on the contrary, no significant difference was observed between the two hemispheric groups in the half space ipsilateral to the lesion.

D) Relationships between contralateral neglect and ipsilateral disorders of attention

If in right brain-damaged patients ipsilateral and contralateral disorders of visual attention were due to destruction of the same attentional neurons, then a significant relationship should exist in these patients between the number of omissions made on the sides of the drawings contralateral and ipsilateral to the brain lesion. This prediction was not confirmed by our results since the correlation coefficient did not reach the level of statistical significance $(r=0 \cdot 29, p$ ns). However, since a mild trend in the expected direction could be seen, a contingency table showing in some detail the relationships existing between ipsilateral and contralateral disorders of attention was constructed in patients with right brain damage.

To do so, both ipsilateral inattention and contralateral neglect were operationally defined as either mild or severe. The mean number of omissions made on the left half of the drawings by patients with right brain damage with contralateral neglect $(5 \cdot 7)$ was used as a cut-off point allowing us to distinguish mild from severe forms of contralateral inattention. Patients with five or less omissions were operationally defined as affected by a mild 
Table 2 Relationships between contralateral neglect and ipsilateral inattention in patients with right brain damage

\begin{tabular}{lrrrrr}
\hline & \multicolumn{5}{c}{ Contralateral Neglect } \\
\cline { 3 - 6 } & & $N^{++}$ & $N^{+}$ & $N^{-}$ & Tot \\
\hline \multirow{3}{*}{ Ipsilateral } & $>1$ & 2 & 2 & 5 & 9 \\
Omissions & 1 & 6 & 5 & 4 & 15 \\
& 0 & 12 & 7 & 59 & 78 \\
& Tot. & 20 & 14 & 68 & 102 \\
\hline
\end{tabular}

N- $\quad=$ No neglect

$\mathrm{N}+\quad=$ Less than six contralateral omissions

$\mathrm{N}++=6$ or more contralateral omissions

degree of contralateral neglect, whereas those with six or more omissions were considered as affected by a severe form of this disorder.

For ipsilateral inattention, only one omission was evaluated as mild, whereas two or more omissions were considered as severe. The relationships between ipsilateral and contralateral disorders of attention are reported in table 2 . Only two out of 20 patients with right brain damage that showed a severe contralateral neglect also showed a severe form of ipsilateral inattention; on the other hand, five out of nine patients with severe ipsilateral inattention did not show any sign of contralateral neglect. These data clearly argue against the hypothesis viewing ipsilateral and contralateral disorders of attention as resulting from damage to the same neuronal population.

E) Relationships between ipsilateral disorders of attention and verbal memory scores in right braindamaged patients

Results of the previous analysis have shown that ipsilateral and contralateral disorders of attention are not due to the same mechanism in patients with right brain damage. Scores obtained on the Rey's 15 words memory test were used to check the alternative hypothesis, viewing ipsilateral disorders as resulting from a widespread lowering of general attention and contralateral neglect as reflecting a specific disorder of visual attention.
To check this hypothesis, we matched results obtained on the Rey's verbal memory test by two groups of patients with right brain damage showing respectively: a) a severe contralateral neglect, in the absence of ipsilateral omissions; b) a severe ipsilateral inattention (as operationally defined in the previous section) with or without contralateral neglect. Results of this analysis are reported in table 3.

These data clearly confirm the hypothesis since they show: a) that patients with severe contralateral neglect but no ipsilateral omissions score significantly better than patients with severe ipsilateral inattention both in immediate and in delayed recall; b) that the vast majority of patients with only contralateral neglect score within the normal range both in immediate and in delayed recall whereas the opposite pattern of results is seen in patients with severe ipsilateral inattention.

\section{Discussion}

The main findings of the present investigation can be summarised as follows: 1) On a drawing completion task, in which patients were requested to complete the missing parts of the designs, right brain-damaged patients failed to complete the contralateral sides of the models significantly more frequently than patients with a left hemisphere injury. On the contrary, no interhemispheric asymmetry was found when the omission of lines lying on the sides of the models ipsilateral to the cerebral lesion was taken into account; 2) No correlation was found between omission of lines lying on the sides of the models contralateral and ipsilateral to the damaged hemispheres; 3 ) Very different results were obtained by patients with right brain damage with severe contralateral neglect but without ipsilateral omission and respectively by subjects with right brain damage with severe ipsilateral inattention on a verbal memory test devised to evaluate the general

Table 3 Results obtained on the Rey's 15 word memory test by patients with right brain damage with severe contralateral neglect but no ipsilateral omissions (Group A) and with more than one ipsilateral omission (Group B)

\begin{tabular}{|c|c|c|c|c|}
\hline \multirow[b]{2}{*}{ Patient number } & \multicolumn{2}{|c|}{ Number of omissions } & \multicolumn{2}{|c|}{ Score obtained on the 15 words } \\
\hline & Contralateral & Ipsilateral & Immediate memory & Delayed memory \\
\hline \multicolumn{5}{|l|}{ GROUP A } \\
\hline 1 & 18 & 0 & 40 & 7 \\
\hline 2 & 16 & 0 & 38 & 7 \\
\hline 3 & 11 & 0 & 37 & 7 \\
\hline 4 & 16 & 0 & 43 & 12 \\
\hline 5 & 10 & 0 & 62 & 14 \\
\hline 6 & 11 & 0 & $31(\mathrm{P})$ & $6(P)$ \\
\hline 7 & 9 & 0 & 47 & 10 \\
\hline 8 & 20 & 0 & 42 & 8 \\
\hline 9 & 6 & 0 & 42 & 10 \\
\hline 10 & 7 & 0 & 55 & 12 \\
\hline 11 & 17 & 0 & 36 & 7 \\
\hline Means & & & $42 \cdot 27$ & 9.09 \\
\hline \multicolumn{5}{|l|}{ GROUP B } \\
\hline 1 & 9 & 5 & $31(P)$ & $5(5)$ \\
\hline 2 & 3 & 2 & $25(P)$ & 1 (P) \\
\hline 3 & 5 & 2 & $26(\mathrm{P})$ & 3 (P) \\
\hline 4 & 3 & 2 & $27(P)$ & $3(P)$ \\
\hline 5 & 4 & 3 & $27(P)$ & $4(\mathrm{P})$ \\
\hline 6 & 5 & 2 & $32(P)$ & $5(P)$ \\
\hline 7 & 4 & 2 & 35 & $0(P)$ \\
\hline 8 & 10 & 5 & $12(\mathrm{P})$ & $0(\mathrm{P})$ \\
\hline 9 & 9 & 5 & $20(P)$ & $3(\mathrm{P})$ \\
\hline Means & & & $26 \cdot 11$ & 2.66 \\
\hline
\end{tabular}

On the Rey's 15 word memory test the cut-off points between normal and pathological performances are set at 35 for the Immediate Memory score and at seven for the Delayed Memory Score $(\mathbf{P})=$ Pathological performance 
attentional resources of the patients. The former scored in general within the normal range whereas the latter obtained a very high number of pathological performances.

Together, these data strongly argue against the assumption that the attention neurons of the right parietal cortex may have partly bilateral receptive fields, whereas the homologous cells of the left parietal cortex have strictly contralateral receptive fields. If this theory was correct the following implications would be predicted:

a) right hemisphere injury should produce not only contralateral neglect but also some inattention for the ipsilateral half space;

b) contralateral neglect and ipsilateral inattention should be highly interrelated, since they result from disruption of the same structure.

Since features of ipsilateral inattention described by Weintraub and Mesulam ${ }^{12}$ could be due to an extension beyond the midline of contralateral neglect, in our research only omissions lying at the extreme lateral part of a pattern were considered as indicative of properly ipsilateral inattention. With this methodological precaution no further difference was observed between patients with right and left brain damage when the omissions of lines lying on the half space ipsilateral to the damaged hemisphere was taken into account.

The second point, which is even more critical, has never been submitted to experimental checking. The results of this study, however, show that no correlation exists between contralateral neglect and ipsilateral inattention, and does not support Heilman's hypothesis but suggests that different kinds of attentional disorders may underlie contralateral and ipsilateral inattention.

The analysis of results obtained on the Rey's 15 word verbal memory test by patients with right brain damage with severe contralateral neglect but no ipsilateral inattention and, respectively, by patients with right brain damage with marked ipsilateral inattention strongly confirmed this suggestion.

Patients with severe contralateral neglect (resulting from a disruption of a specific component of visual attention) scored within the normal range on the verbal memory test. By contrast, patients with marked ipsilateral inattention obtained very low scores on the same test, showing that the inability to pay attention to the half space ipsilateral to the brain damage is part of a diffuse and non specific reduction of the attentional resources of the patient.

1 Brain WR. Visual disorientation with special reference to lesions of the right hemisphere. Brain 1941;64:244-72.

2 Colombo A, De Renzi E, Faglioni P. The occurrence of visual neglect in patients with unilateral cerebral disease. Cortex 1976;12:221-31.

3 De Renzi E. Disorders of space exploration and cognition. Chichester: Wiley, 1982 .

4 De Renzi E, Faglioni P, Scotti G. Hemispheric contribution to exploration of space through the visual and tactile modality. Cortex 1970;6:191-203.

5 Gainotti G. Les manifestations de negligence et d'inattention pour l'hemispace. Cortex 1968;4:64-91.

6 Gainotti G, Messerli P, Tissot R. Qualitative analysis of unilateral spatial neglect in relation to laterality of cerebral unilateral spatial neglect in relation to laterality of cerebra

lesions. J Neurol Neurosurg Psychiatry 1972;35:545-50.
7 Hecaen H. Introduction a la Neuropsychologie. Paris: Larousse, 1972.

8 Heilman KM. Neglect and related disorders. In: Heilman $\mathrm{KM}$ and Valenstein E, eds. Clin Neuropsychol. New York: Oxford University Press, 1979.

9 Heilman KM, Valenstein E. Mechanisms underlying hemispatial neglect. Ann Neurol 1979;5:166-70.

10 Heilman KM, Valenstein E, Watson AT. The neglec syndrome. In: Vinken PJ, Bruyn GV, Klawans HL, eds. Handbook of Clinical Neurology. Amsterdam: Elsevier Science Publisher, 1985.

11 Heilman KW, Van Den Abell T. Right hemispheric dominance for attention: the mechanism underlying hemispheric
asymmetries of inattention (neglect). Neurology 1980;30:327-30.

12 Weintraub S, Mesulam MM. Right cerebral dominance in spatial attention: further evidence based on ipsilateral neglect. Arch Neurol 1987;44:621-5.

13 Gainotti G, De Bonis C, Daniele A, Caltagirone C. Contralateral and ipsilateral tactile extinctions in patients with right and left brain damage. Int $J$ Neurosci 1989;45:81-9.

14 Rey A. Memorisation d'une serie de 15 mots en 5 repetitions In: Rey A, ed. L'examen clinique en psychologie. Paris: Pres Universitaires de France, 1958.

15 Caltagirone C, Gainotti G, Masullo C, Miceli G. Validity of some neuropsychological tests in the mental assessment of mental deterioration. Acta Psychiatrica Scandinavica 1979;60:50-6. 